\title{
A Scalable Empirical Bayes Approach to Variable Selection in Generalized Linear Models
}

\author{
Haim Y. Bar* \\ Department of Statistics University of Connecticut, \\ Storrs CT, USA. \\ and \\ James G. Booth and Martin T. Wells \\ Department of Statistics and Data Science, Cornell University, \\ Ithaca NY, 14853, USA.
}

October 23, 2019

\begin{abstract}
A new empirical Bayes approach to variable selection in the context of generalized linear models is developed. The proposed algorithm scales to situations in which the number of putative explanatory variables is very large, possibly much larger than the number of responses. The coefficients in the linear predictor are modeled as a three-component mixture allowing the explanatory variables to have a random positive effect on the response, a random negative effect, or no effect. A key assumption is that only a small (but unknown) fraction of the candidate variables have a non-zero effect. This assumption, in addition to treating the coefficients as random effects facilitates an approach that is computationally efficient. In particular, the number of parameters that have to be estimated is small, and remains constant regardless of the number of explanatory variables. The model parameters are estimated using a Generalized Alternating Maximization algorithm which is scalable, and leads to significantly faster convergence compared with simulation-based fully Bayesian methods.
\end{abstract}

Keywords: Feature selection; Generalized linear mixed model; High dimensional data; EM algorithm; Mixture model; Sparsity

*The authors gratefully acknowledge the following funding support: Prof. Bar's research was supported by NSF-DMS 1612625. Professor Booth's research was partially supported by an NSF grant, nsfdms 1208488, and by nsf-dms 1611893 . Professor Wells' research was partially supported by nsf-dms 1208488, nsf-dms 1611893, and NIH grant U19 AI111143. 


\section{Introduction}

This paper concerns variable selection in generalized linear regression models when there are a large number of candidate explanatory variables (putative predictors), most of which have little or no effect on the dependent variable. An empirical Bayes, modelbased approach is proposed that is implemented via a fast and scalable Generalized Alternating Maximization algorithm.

The new age of high speed computing and technological advances in genetics and molecular biology, for example, have dramatically changed modeling and computation needs. It is now common for researchers to want to estimate the effects of hundreds or even thousands of predictors $(K)$ on a given response, often with a far smaller sample size $(N)$. In such cases, traditional fitting methods such as least squares break down. In addition, even with a relatively modest number of predictors, the model space can be large enough to render exhaustive search-based algorithms impractical.

Automated methods for variable selection in normal linear regression models have long been studied in the literature; see, for example Hocking (1976). Nowadays virtually every statistical package contains an implementation of standard stepwise methods that typically add or remove one variable from the model in each iteration, based on sequential F-tests and a threshold, or a well-known selection criterion such as AIC , BIC, or Mallow's- $C_{p}$. A modern alternative is to use false discovery rate (FDR) for stepwise model selection (Benjamini and Gavrilov, 2009).

Much of the recent literature has focused on variations of penalized likelihood approaches in which coefficient estimation and variable selection are done simultaneously. The most well-known method of this type is the LASSO (Tibshirani, 1996) which minimizes the residuals sum of squares subject to an $\ell_{1}$ constraint. This constraint allows the number of non-zero parameter estimates to be controlled and adapt to sparsity. Other related methods that are based on a minimizing a loss function, subject to a constraint on the complexity of the model, include SCAD (Fan and Li, 2001), the adaptive LASSO (Zou, 2006), LARS (Efron et al., 2004; Hesterberg et al., 2008), and more recent proposals by Bogdan et al. (2014), Bühlmann et al. (2014) and Lederer and Müller (2015).

Bayesian approaches are another important direction in model selection research. 
Significant contributions include George and McCulloch (1993), Casella and Moreno (2006), and the spike-and-slab method in Ishwaran and Rao (2005). The model proposed here is similar to Zhang et al. (2005), and Guan and Stephens (2012) whose work is motivated by QTL and genome-wide association studies (GWAS). Our model allows for a fully-Bayesian implementation, but an empirical Bayes analysis via the Generalized Alternating Maximization algorithm (Gunawardana and Byrne, 2005) is proposed instead because the running time of an MCMC sampler is too long for many data sets in modern applications. For example, in our simulations (Section 4) we find that the faster of two MCMC-based variable selection methods required 40 minutes to complete 1000 MCMC iterations. In contrast, non-MCMC methods required only a few seconds to perform variable selection, and generally, gave better results. In this sense the algorithm is a close competitor to the exact EM algorithm of Rǒcková and George (2014) based on a Bayesian spike-and-slab model. A key advantage of the approach proposed in this paper is that it extends in a straightforward manner to the generalized linear model framework.

Recently developed continuous prior distributions have proven more effective in sparse regression than the a Bayesian lasso (Park and Casella, 2008) that uses a Laplace prior, which fails to simultaneously induce sparsity while efficiently recovering nonnull parameters (van der Pas et al., 2016). Among these are "global-local priors" including the Horseshoe-type priors (Carvalho et al., 2010; Bhadra et al., 2017) and the Gamma Gamma prior (Bai and Ghosh, 2017). These approaches offer computational advantages relative to two-component spike-and-slab mixture priors since one does not need to explore a complex discrete model space of size $2^{K}$. A limitation of the continuous shrinkage prior approach is that it fails to provide a sparse solution. To address the problem, ad hoc post-processing methods for producing sparse estimates from posterior samples that decoupled shrinkage and selection have been developed (Hahn and Carvalho, 2015).

The remainder of the article is organized as follows. The model and notation are introduced in Section 2. A Generalized Alternating Maximization fitting algorithm and selection procedure is described in Section 3 Section 4 describes results of simulation 
studies in which the proposed procedure is compared with several variable selection packages, including ncvreg (Breheny and Huang, 2011) and SIS (Saldana and Feng, 2018), both of which implement three types of penalties: LASSO (Tibshirani, 1996), SCAD (Fan and Li, 2001), and MCP (Zhang, 2010). Other packages we included in our simulations are: glmnet (Friedman et al., 2010), lars (Hastie and Efron, 2013), EMVS (Rǒcková and Moran, 2018), spikes lab (Ishwaran et al., 2013), mombf (Rossell et al., 2018), and BoomSpikeSlab (Scott, 2017). We also compared the performance of our method with a one-predictor-at-a-time approach, controlling the false discovery rate (Benjamini and Hochberg, 1995). In Section 5 we discuss some applications. First, we demonstrate our method when the response is normal (the logarithm of vitamin B12 production rate). In this case the sample size is $N=71$ and the number of predictors is 4,088 . We compare the model obtained from our method with ones obtained from TREX (Lederer and Müller, 2015), and the results obtained by Bühlmann et al. (2014). The second example demonstrates an application to a binary response (categorized Body Mass Index), which we compare to an analysis using the continuous outcome. In this case, $N=96$ and there are 45 (compositional) predictors. Finally, we explain how to perform variable selection in survival analysis by treating the number of deaths in a sequence of non-overlapping time intervals as a Poisson counts. The paper concludes with with some discussion in Section 6. Important implementation considerations and additional examples are discussed in the supplementary material.

\section{A Statistical Model for Automatic Variable Selection}

Consider responses $y_{i}, i=1, \ldots, N$, and assume that the mean of the $i$ th response, $\lambda_{i}$, is linked to a linear predictor, $\eta_{i}$, as follows:

$$
g\left(\lambda_{i}\right) \equiv \eta_{i}=\sum_{j=1}^{J} x_{i j} \beta_{j}+\sum_{k=1}^{K} z_{i k} \gamma_{k} u_{k}
$$

The model (1) allows for $J \geq 0$ predictors, $x_{i j}$, that are always included in the model and a set of $K>0$ 'putative' predictors, $z_{i k}$, from which it is expected only a small 
subset are to be included. Here, $\beta_{j}$ is the coefficient associated with the $j$ th 'lockedin' predictor, $u_{k}$ is a random coefficient associated with the $k$ th putative predictor. We assume that each $\gamma_{k} u_{k}$ belongs to one of three components, $C_{L}, C_{0}$, and $C_{R}$, such that $\gamma_{k}=0$ for $k \in C_{0}, \gamma_{k}=-1$ for $k \in C_{L}$, and $\gamma_{k}=1$ for $k \in C_{R}$. We also assume that $u_{k} \stackrel{\text { iid }}{\sim} N\left(\mu, \sigma^{2}\right)$, independently of $\gamma_{k}$. Justification for both of these choices is given in Section 3 . Thus, the inclusion of the $k$ th predictor in the linear model is determined by the value of $\gamma_{k} \stackrel{\text { iid }}{\sim}$ multinomial $\left(-1,0,1 ; p_{L}, p_{0}, p_{R}\right)$. Specification of the distribution of the responses is completed by assuming that, conditional on the linear predictors, they are independent draws from a particular exponential dispersion family, with the most important special cases being the normal, binomial and Poisson distributions. Within this modeling framework the problem of variable selection is cast as a classification problem, for which the main interest lies in identifying which putative variables belong to $C_{L} \cup C_{R}$, i.e., which latent variables, $\gamma_{k}$, are non-zero.

Let $\mathbf{X}$ denote the $N \times J$ matrix with $j$ th column, $\mathbf{x}_{j}$, containing the values of the $j$ th 'locked in' predictor. Similarly, define $\mathbf{Z}$ to be the $N \times K$ matrix with $k$ th column $\mathbf{z}_{k}$, the corresponding vector for the $k$ th putative predictor. Then the linear predictor model (1) can be rewritten in matrix form as

$$
\eta=\mathbf{X} \beta+\mathbf{Z} \mathbf{\Gamma u}
$$

where $\boldsymbol{\beta}=\left(\beta_{1}, \ldots, \beta_{J}\right)^{\prime}, \boldsymbol{\Gamma} \equiv \operatorname{diag}\left(\gamma_{1}, \gamma_{2}, \ldots, \gamma_{K}\right)$. Furthermore, the distributional assumptions concerning $\left\{u_{k}\right\}$ imply

$$
\mathbf{Z} \mathbf{\Gamma} \mathbf{u} \mathbf{\Gamma} \sim N\left(\mathbf{Z} \boldsymbol{\Gamma} \boldsymbol{\mu}, \sigma^{2} \mathbf{Z} \boldsymbol{\Gamma}^{2} \mathbf{Z}^{\prime}\right)
$$

where $\boldsymbol{\mu}=\mathbf{1}_{K} \mu$.

Mixture models provide simplicity and tractability and are very popular in many applications. However, they are known to have undesirable mathematical properties, such as unbounded likelihood and lack of identifiability (Chen and Li, 2009;: McLachlan and Peel, 2000). For example, in the Gaussian case, if $\mu=0$ then the product $\gamma_{k} u_{k}$ in model (1) has marginal density of the form $h(x)=p_{0} \cdot 0+\left(p_{L}+p_{R}\right) \varphi(x ; 0, \sigma)$, where $\varphi(\cdot ; \mu, \sigma)$ 

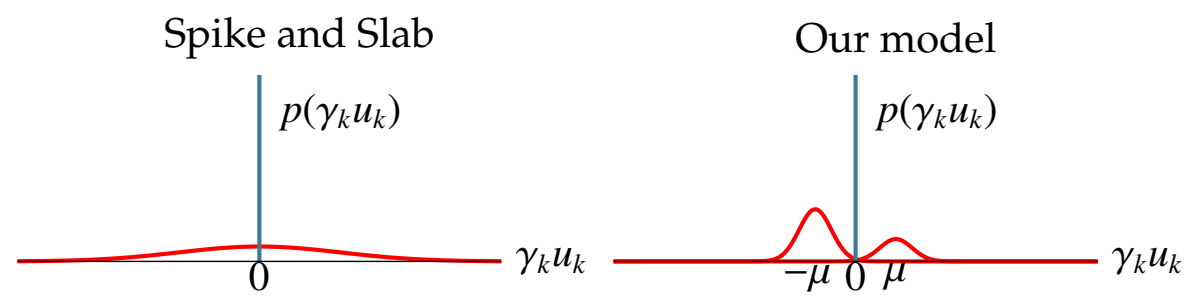

Figure 1: A graphical representation of the spike and slab model vs. our mixture model.

denotes a normal density. Clearly, $p_{L}$ and $p_{R}$ cannot be separately identified in such a model. However, when $\mu \neq 0$, the density is $h(x)=p_{0} \cdot 0+p_{L} \varphi(x ;-\mu, \sigma)+p_{R} \varphi(x ; \mu, \sigma)$, which is identifiable if $p_{L} \neq p_{R}$. Moreover, even if $p_{L}=p_{R}$, the identifiability issue only concerns the sign of $\mu$ which, in this case, does not affect the marginal distribution $h$.

Our hierarchical mixture model is similar to the well-known Spike and Slab model of Ishwaran and Rao (2005) which is implemented, for example, in the R package BoomSpikeSlab (Scott, 2017) and in EMVS (Rǒcková and George, 2014). The main difference is our choice of a three-way mixture model, in which there are two non-null components, rather than one. Compared with the spike and slab approach, our model offers a couple of advantages. To illustrate these advantages, it is helpful to plot the theoretical distributions of $\gamma_{k} u_{k}$ under the two models (Fig. 11). The two-component mixture is depicted on the left, and our model on the right. In both cases, $p_{0}=0.8$.

The two-component mixture assumes that the non-null distribution is symmetric, which implies a prior belief that the proportion of variables which are positively correlated with the response is the same as the proportion of predictors which are negatively correlated with the response. This may be an unreasonable assumption, and the threecomponent mixture model is more flexible in this regard.

In addition, the non-null component in the two-component mixture has much of its mass around zero, which is counterintuitive because it is assumed that variables in the non-null component have a non-zero effect. In contrast, the three-component model assigns a very small probability to non-null values near zero. Our mixture model also allows for the non-null components to be highly concentrated, which may be especially useful in situation where there is a single significant predictor. The assumption of symmetry of the two nonnull components in our model may be relaxed, but it has some 
important benefits. First, it implies that the model is invariant to the sign of columns of $\mathbf{Z}$. Second, it allows us to borrow information across the two nonnull components, which greatly contributes to computational stability, as well as power to detect true predictors, and maintaining a low false positive rate. This is especially beneficial when at least one of the nonnull components consists of a small number of predictors.

The three component prior can be viewed as similar to a non-local prior for which density functions are identically zero whenever a model parameter is equal to its null value. Conversely, spike and slab priors are local priors where component densities are positive at null parameter values. Johnson and Rossell (2010, 2012) demonstrate that model selection procedures based on non-local prior densities assign a posterior probability of one to the true model as the sample size $n$ increases and certain regularity conditions on the design matrix pertain. Furthermore under the same conditions, they show that standard approaches based on local prior specifications result in the asymptotic assignment of a posterior probability of zero to the true model.

\section{Estimation and Variable Selection}

\subsection{The Complete Data Likelihood}

The marginal distribution of the responses according to the model described in the previous section is determined by the parameter vector $\boldsymbol{\theta}=\left\{\boldsymbol{\beta}, \mu, \sigma^{2}, \boldsymbol{p}, \phi\right\}$, where $\phi$ is a dispersion parameter which, depending on the GLM specification, may or may not be known. Define $n_{j}=\sum_{k=1}^{K} I\left(\gamma_{k}=j\right)$, for $j=-1,0,1$ corresponding to the left, middle, and right components of the mixture model, respectively, and let $\varphi(\cdot ; \mu, \Sigma)$ denote a multivariate normal density with mean $\boldsymbol{\mu}$ and covariance matrix $\boldsymbol{\Sigma}$. Then assuming a canonical link function, the complete data log likelihood is

$$
\begin{aligned}
\ell(\mathbf{y}, \boldsymbol{\gamma} \mid \boldsymbol{\theta})=\log f_{C}(\mathbf{y}, \boldsymbol{\gamma} \mid \boldsymbol{\theta})= & \log \int \exp \left\{\sum_{i=1}^{N} \frac{m_{i}}{\phi}\left[\eta_{i} y_{i}-b\left(\eta_{i}\right)\right]\right\} \varphi\left(\mathbf{u} ; \boldsymbol{\Gamma} \boldsymbol{\mu}, \sigma^{2} \mathbf{Z} \boldsymbol{\Gamma}^{2} \mathbf{Z}^{\prime}\right) d \mathbf{u} \\
& +\sum_{i=1}^{N} c\left(y_{i}, \phi / m_{i}\right)+\sum_{j=-1,0,1} n_{j} \log p_{j},
\end{aligned}
$$


where the $m_{i}$ 's are known positive weights, $b$ is the cumulant generator for the GLM satisfying $b^{\prime}\left(\eta_{i}\right)=\lambda_{i}$, and $b^{\prime \prime}\left(\eta_{i}\right)=V\left(\lambda_{i}\right)$, where $V$ is the GLM variance function.

In the Gaussian case the integral in (2) has a closed form and the complete data loglikelihood reduces to

$$
\begin{aligned}
\ell(\mathbf{y}, \boldsymbol{\gamma} \mid \boldsymbol{\theta})= & -\frac{1}{2}(\mathbf{y}-\mathbf{X} \boldsymbol{\beta}-\mathbf{Z} \boldsymbol{\Gamma} \boldsymbol{\mu})^{\prime}\left(\phi \mathbf{W}^{-1}+\sigma^{2} \mathbf{Z} \boldsymbol{\Gamma}^{2} \mathbf{Z}^{\prime}\right)^{-1}(\mathbf{y}-\mathbf{X} \boldsymbol{\beta}-\mathbf{Z} \boldsymbol{\Gamma} \boldsymbol{\mu}) \\
& -\frac{1}{2} \log \left|\phi \mathbf{W}^{-1}+\sigma^{2} \mathbf{Z} \boldsymbol{\Gamma}^{2} \mathbf{Z}^{\prime}\right|+\sum_{j=-1,0,1} n_{j} \log p_{j}-\frac{N \log (2 \pi)}{2},
\end{aligned}
$$

where $\mathbf{W}=\operatorname{diag}\left(w_{i}\right)$ and $w_{i} \equiv m_{i}$. For non-Gaussian GLMs an approximate complete data log-likelihood is obtained by substituting the so-called 'working response' and 'iterative weight matrix' in place of $\mathbf{y}$ and $\mathbf{W}$ in (3), with components given by

$$
\tilde{y}_{i}=g\left(\tilde{\lambda}_{i}\right)+g^{\prime}\left(\tilde{\lambda}_{i}\right)\left(y_{i}-\tilde{\lambda}_{i}\right) \text { and } \tilde{w}_{i}=m_{i} / g^{\prime}\left(\tilde{\lambda}_{i}\right) \text {. }
$$

The substitutions can be justified on the basis of a Laplace approximation to the integral in (2), and are the basis of numerous algorithms in the literature for fitting GLMs with random effects. See, for example, Schall (1991); Breslow and Clayton (1993); Wolfinger and O'Connell (1993); McGilchrist (1994).

\subsection{A Generalized Alternating Maximization Algorithm}

In principle, the ML estimate of the model parameter vector, $\boldsymbol{\theta}$, can be obtained using the EM algorithm (Dempster et al., 1977) using the complete data log-likelihood given in (3), with the Q-function given by $Q\left(\boldsymbol{\theta} ; \boldsymbol{\theta}^{\prime}\right)=E_{\boldsymbol{\theta}^{\prime}}\left\{\log f_{c}(\mathbf{y}, \boldsymbol{\gamma} \mid \boldsymbol{\theta}) \mid \mathbf{y}\right\}$ where $\boldsymbol{\theta}^{\prime}$ denotes the current estimate of $\boldsymbol{\theta}$. However, in this case the expectation is intractable, so we propose using the simple plug-in approximation

$$
E_{\boldsymbol{\theta}^{\prime}}\left\{\log f_{c}(\mathbf{y}, \boldsymbol{\gamma} \mid \boldsymbol{\theta}) \mid \mathbf{y}\right\} \approx E_{\boldsymbol{\theta}^{\prime}}\left\{\log f_{c}\left(\mathbf{y}, \boldsymbol{\gamma}^{\prime} \mid \boldsymbol{\theta}\right) \mid \mathbf{y}\right\}
$$

where $\gamma^{\prime}$ is obtained using one of the methods described later in this section.

Since the expectation terms are approximated, the resulting iterative estimation pro- 
cedure will not fall within the EM framework, or even within the GEM framework ( $\mathrm{Wu}$, 1983), and thus, convergence results from these frameworks will not apply. This type of EM variant, where the E-step is replaced with an approximation is an example of the Generalized Alternating Minimization (GAM) framework of Gunawardana and Byrne (2005), of which EM and GEM are special cases. We will use the GAM theory to show the convergence of our algorithm, which we describe in Algorithm 1 .

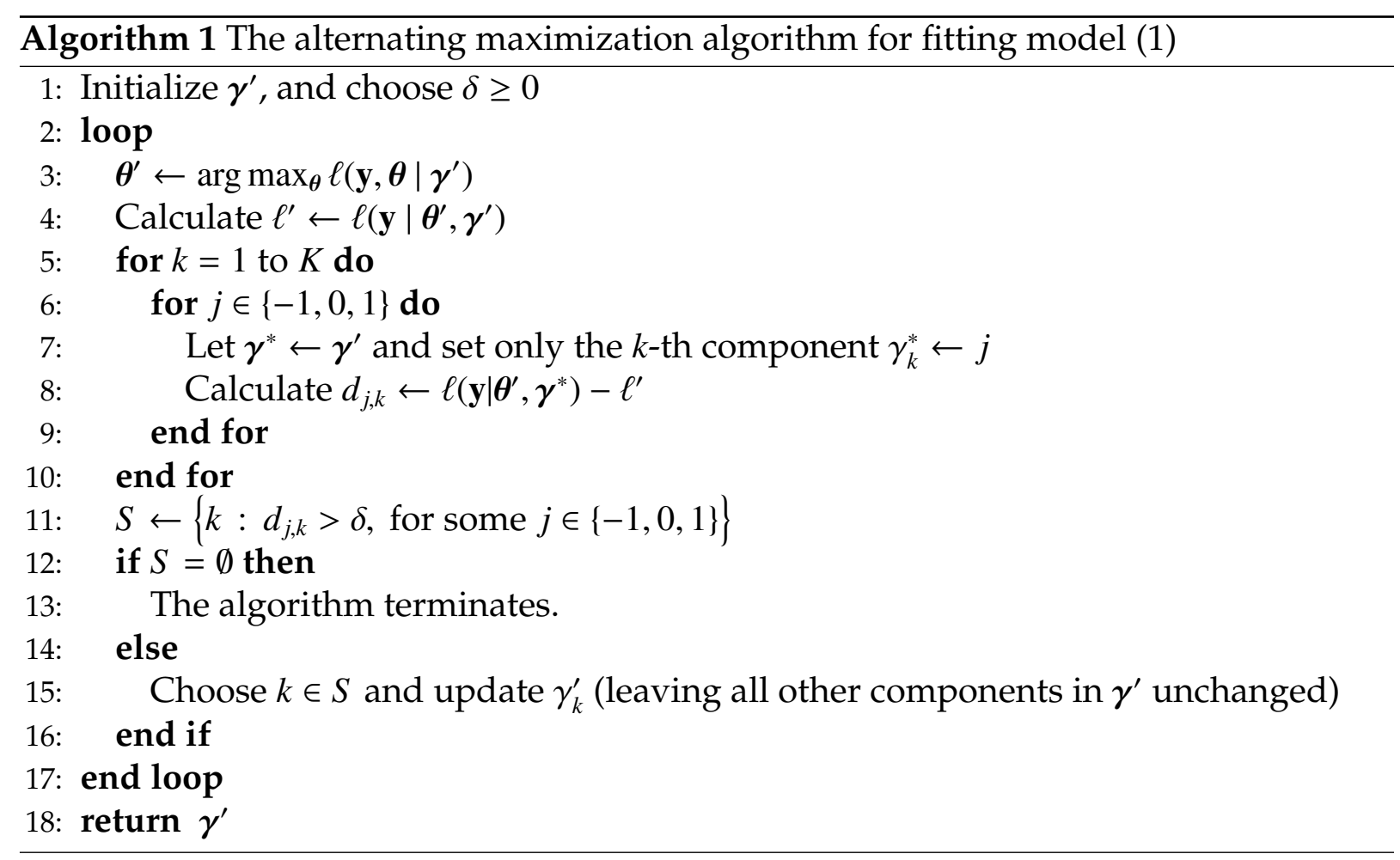

This algorithm uses a 'likelihood-ratio' approach because a latent variable $\gamma_{k}$ is changed from its current value if that change increases the likelihood in a meaningful way (holding all other latent indicators at their current values). The set $S$ consists of all the variables which yield an improvement greater than $\delta$ in the loglikelihood when their current classification according to the three-component mixture model is changed, while holding all other $\gamma_{k} \mathrm{~s}$ at their current values. If $S$ is not empty, choosing $k$ from this set in Line 15 is done according to one of the following methods:

- Greedy: choose $k \in S$ for which $d_{j, k}$ is largest. 
- Weighted probability: choose $k \in S$ with probability

$$
\frac{d_{j, k}}{\sum_{(r, s) \in S} d_{r, S}} .
$$

Proposition 1 For the model in (2) Algorithm 1 converges to a stationary point in a finite number of steps.

A proof of the proposition is given in Appendix $\mathrm{A}$

\subsection{Notes and Further Details}

First, recall that in the binomial and Poisson models we work with the 'working response and weights', so $\tilde{y}_{i}$ and $\tilde{w}_{i}$ have to be iteratively updated at each iteration of Algorithm 1.

Second, we emphasize that our algorithm modifies at most one coordinate in each iteration because, changing more than one variable may introduce multicollinearity. What this means is that even though each single variable may increase the likelihood, changing a set of variables which are highly correlated may cause the log-likelihood to decrease (and it may even approach $-\infty$, because $\ell$ involves the logarithm of the precision matrix.) As a consequence, our algorithm automatically prevents selecting models with highly correlated predictors. We do, however, keep track of predictors that are correlated with ones selected to be in the model, since they are likely to be related to the outcome as well. We return to this point in the Case Studies section.

Third, we may choose $\gamma^{\prime}$ in Line 1 to be the variables selected by any other method (FDR, SIS, EMVS, etc.) Then, the log-likelihood of the final model selected by our algorithm will be greater than or equal to the one obtained at the $0^{\text {th }}$ iteration.

Fourth, as a referee pointed out, the parameter space for $(\boldsymbol{\theta}, \boldsymbol{\gamma})$ cannot assumed to be unimodal, especially when some putative variables are correlated with each other or with columns in $\mathbf{X}$. Therefore, it is recommended to run Algorithm 1 1 multiple times, using the weighted probability approach for selecting the next putative variable to be updated. This approach is feasible because our method is computationally efficient. When the posterior distribution is multimodal there is no one 'correct' model, and running 
the algorithm multiple times will allow users to obtain different, but possibly equally relevant sets of significant predictors each time they fit the model. This approach is demonstrated in Section 5 (the riboflavin data example).

Finally, to obtain the maximum likelihood estimates $\boldsymbol{\theta}^{\prime}$ in Line 3 , the following formulas are used. Let $\boldsymbol{\Sigma}=\phi \mathbf{W}^{-1}+\sigma^{2} \mathbf{Z} \Gamma^{2} \mathbf{Z}^{\prime}$ and $\mathbf{H}=[\mathbf{X}, \mathbf{Z} \Gamma \mathbf{1}]$, the update formula for $\tilde{\boldsymbol{\beta}}=\left(\boldsymbol{\beta}^{\prime}, \mu\right)^{\prime}$ is given by

$$
\tilde{\boldsymbol{\beta}}=\left(\mathbf{H}^{\prime} \boldsymbol{\Sigma}^{-1} \mathbf{H}\right)^{-1} \mathbf{H}^{\prime} \boldsymbol{\Sigma}^{-1} \mathbf{y}
$$

so $\mu$ is simply the last element in $\tilde{\boldsymbol{\beta}}$, and the updates for the variance components are given by

$$
\phi=\frac{\tau_{e}}{N} \quad \text { and } \quad \sigma^{2}=\frac{\tau_{r}}{\operatorname{rank}(\mathbf{Z \Gamma})}
$$

provided $\operatorname{rank}(\mathbf{Z \Gamma})>0$ and $\mu=\sigma^{2}=0$ otherwise, where

$$
\begin{aligned}
\tau_{e} & =\operatorname{trace}\left[\phi \mathbf{I}_{N}-\phi^{2} \boldsymbol{\Sigma}^{-1}\right]+\phi^{2}(\mathbf{y}-\mathbf{H} \tilde{\boldsymbol{\beta}})^{\prime} \boldsymbol{\Sigma}^{-2}(\mathbf{y}-\mathbf{H} \tilde{\boldsymbol{\beta}}) \\
\tau_{r} & =\operatorname{trace}\left[\sigma^{2} \mathbf{I}_{K}-\sigma^{4} \boldsymbol{\Gamma} \mathbf{Z}^{\prime} \boldsymbol{\Sigma}^{-1} \mathbf{Z} \boldsymbol{\Gamma}\right]+\sigma^{4}(\mathbf{y}-\mathbf{H} \tilde{\boldsymbol{\beta}})^{\prime} \boldsymbol{\Sigma}^{-1} \mathbf{Z} \boldsymbol{\Gamma}^{2} \mathbf{Z}^{\prime} \boldsymbol{\Sigma}^{-1}(\mathbf{y}-\mathbf{H} \tilde{\boldsymbol{\beta}}) .
\end{aligned}
$$

(see Section 8.3.b in Searle et al. 1992). Finally, maximizing (3) with respect $p_{L}, p_{0}, p_{R}$ leads to the updates $p_{j}=n_{j} / K$ for $j=-1,0,1$.

Small values of $\mu$ are counterintuitive because they suggest that the mean effects of selected putative variables are close to zero. Moreover, as noted earlier, small values of $\mu$ can lead to identifiability problems. To prevent such problems one might consider adding a penalty term on $\mu$ as proposed by Chen and Li (2009). Another possible approach to preventing identifiability problems is to use a different nonnull prior, such as a mixture of two log-normal distributions, so that the probability that variables with effect size close to zero will be considered as nonnull, will be practically 0 . However, as we shall see in the next subsection, one of the strengths of our method is that it allows us to achieve significant dimension reduction via the Woodbury identity, which requires the normality assumption. So, were we to use a different nonnull distribution, we would 
need to add an extra step to the algorithm in order to normalize the nonnull components (via a Laplace approximation, for example). In practice, however, we find that identifiability problems are effectively avoided by choosing appropriate initial values for our Generalized Alternating Maximization algorithm. For further details regarding the initialization of the algorithm, see the Supplementary Material.

\subsection{Modifications for large $N$ and $K$}

The complete data log-likelihood (2) contains a large $(N \times N)$ matrix which has to be inverted to compute the iterative approximate ML estimates. However, using the Woodbury identity (Golub and Van Loan, 1996),

$$
\begin{aligned}
\boldsymbol{\Sigma}^{-1} & =\left(\phi \mathbf{W}^{-1}+\sigma^{2} \mathbf{Z} \Gamma^{2} \mathbf{Z}^{\prime}\right)^{-1} \\
& =\frac{1}{\phi}\left[\mathbf{W}^{\frac{1}{2}}\left(\mathbf{I}_{N}+\frac{\sigma^{2}}{\phi} \mathbf{W}^{\frac{1}{2}} \mathbf{Z} \boldsymbol{\Gamma}^{2} \mathbf{Z}^{\prime} \mathbf{W}^{\frac{1}{2}}\right)^{-1} \mathbf{W}^{\frac{1}{2}}\right] \\
& =\frac{1}{\phi}\left[\mathbf{W}-\frac{\sigma^{2}}{\phi} \mathbf{W}^{\frac{1}{2}} \mathbf{Z} \boldsymbol{\Gamma}^{\prime}\left(\mathbf{I}_{K}+\frac{\sigma^{2}}{\phi} \boldsymbol{\Gamma}^{\prime} \mathbf{Z}^{\prime} \mathbf{W} \mathbf{Z} \boldsymbol{\Gamma}\right)^{-1} \boldsymbol{\Gamma}^{\prime} \mathbf{Z}^{\prime} \mathbf{W}^{\frac{1}{2}}\right] .
\end{aligned}
$$

This simplifies the computations considerably because the $(k, l) t h$ element of $\boldsymbol{\Gamma}^{\prime} \mathbf{Z}^{\prime} \mathbf{W Z \Gamma}$ is proportional to $\gamma_{k} \gamma_{l}$. Specifically, suppose there are $L$ variables for which $\gamma_{k} \neq 0$. Define $\boldsymbol{\Gamma}_{L}$ to be the $L \times L$ reduced matrix in which rows and columns of $\boldsymbol{\Gamma}$ corresponding to excluded putative variables have been eliminated. Similarly, define $\mathbf{Z}_{L}$ by eliminating the corresponding rows of $\mathbf{Z}$. Then

$$
\boldsymbol{\Sigma}^{-1}=\frac{1}{\phi}\left[\mathbf{W}-\frac{\sigma^{2}}{\phi} \mathbf{W}^{1 / 2} \mathbf{Z}_{L} \boldsymbol{\Gamma}_{L}^{\prime}\left(\mathbf{I}_{L}+\frac{\sigma^{2}}{\phi} \boldsymbol{\Gamma}_{L}^{\prime} \mathbf{Z}_{L}^{\prime} \mathbf{W} \mathbf{Z}_{L} \boldsymbol{\Gamma}_{L}\right)^{-1} \boldsymbol{\Gamma}_{L}^{\prime} \mathbf{Z}_{L}^{\prime} \mathbf{W}^{\frac{1}{2}}\right] .
$$

Thus, inversion of the $N \times N$ matrix $\Sigma$ is reduced to inverting a much lower dimensional $L \times L$ matrix. Similar simplifications, due to the exclusion of most putative variables, apply to computation of the determinant term in (3).

Further details about implementation considerations are provided in the Supplementary Materials. Specifically, we discuss how to deal with correlation and interactions among the putative variables, and we discuss computational challenges stemming 
from the large number of variables, such as how to avoid loading the entire matrix $\mathbf{Z}$ to memory, and how to parallelize the algorithm.

\section{Simulations}

We conducted a simulation study to verify that under the assumed model (1) the algorithm yields accurate parameter estimates (not shown here), and to compare the performance of our algorithm in terms of power and accuracy with other methods. Not surprisingly, as the sample size increases (even when $N$ is still much smaller than $K$ ), the parameter estimates become more accurate, the power to detect the non-null variables increases, and the Type-I error rate decreases.

In this section we focus on the results of a simulation study that compares the Type I and Type II errors when the data are not necessarily generated according to model (1). We call the program that implements variable selection according to model (1) SEMMS (Scalable EMpirical Bayes Model Selection), and compare its performance with several well-known variable selection approaches:

- ncvreg (Breheny and Huang, 2011), which implements three types of penalties: LASSO (Tibshirani, 1996), SCAD (Fan and Li, 2001), and MCP (Zhang, 2010).

- glmnet (Friedman et al., 2010), to fit GLM models with the lasso or elastic net regularization.

- lars (least angle regression) (Hastie and Efron, 2013).

- sIs Sure Independence Screening (Saldana and Feng, 2018). This package combines a screening process which reduces the number of variables to be considered in each regularization step, while ensuring that only irrelevant predictors are eliminated. This package allows to choose among three penalty types (LASSO, SCAD, or $\mathrm{MCP})$.

- EMVS (Rǒcková and Moran, 2018), a Bayesian approach to variable selection with a fast implementation via the EM algorithm.

- spikeslab (Ishwaran et al., 2013), a Bayesian (spike and slab) variable selection method. 
- mombf (Rossell et al. 2018) a Bayesian (MCMC) method based on Johnson and Rossell (2012) using moment and inverse moment Bayes Factors.

- Boomspikeslab (Scott, 2017), a Bayesian (MCMC) implementation of the spike and slab model.

- FDR (Benjamini and Hochberg, 1995) a simple one-predictor-at-a-time approach, controlling the false discovery rate. In our simulations, we controlled the FDR at the 0.05 level.

The comparison with spikeslab and EMVS is only done in the normal response case, since these packages do not have an option to fit a GLM model. Similarly, lars only handles a normal linear regression model, but the package covTest (Lockhart et al. (2013) includes the function lars . glm which can also be used to analyze binomial response and Cox regression models (but not Poisson).

Generally, these software packages were used with their default values. With lars and glmnet, the number of selected variables was such that together they explained $90 \%$ of the variability or (null) deviance. The packages ncvreg and SIS simply return the selected variables. When using spikes lab we set the 'bigp.smalln' option to TRUE, and the selected variables were the non-zero generalized elastic net (gnet) coefficients. With EMVS we varied the spike variance parameter (twenty values between $10^{-10}$ and $10^{-1}$ equally spaced on the logarithmic scale) and the type of the prior distribution was set to 'betabinomial'. The 'independent' parameter was set to FALSE, since it yielded much better results than the default (independent=TRUE, which resulted in zero selected predictors in most cases.) Hence, rather than running EMVS under the assumption that the regression coefficients and the error variance are independent, a priori, a conjugate prior was used (Rǒcková and George, 2014).

In the case of a normal response we show results from nine different scenarios with varying dependence structures and number of significant predictors. In each scenario $K=1000$ predictors were initially drawn independently from a Unif $[-1,1]$ distribution, but in scenarios 3, 4, and 5 they were modified in order to induce correlations. Scenarios 1-5 are made under the assumption of our mixture model, while 6-9 are not. The true number of predictors in each scenario is denoted by L. The error terms, $\epsilon_{i}$, are generated 
as i.i.d. $N(0,0.1)$ variates, except for $\mathrm{N} 2$ where $\epsilon_{i} \sim N(0,0.25)$.

(N1) A single significant predictor $(L=1)$ is related to the response: $Y_{i}=Z_{1 i}+\epsilon_{i}$.

(N2) The response is the sum of eight i.i.d. predictors $(L=8): Y_{i}=Z_{1 i}+\ldots+Z_{8 i}+\epsilon_{i}$.

(N3) $Y_{i}=Z_{1 i}+\ldots+Z_{8 i}+\epsilon_{i}($ again, $L=8)$ but $Z_{2}=Z_{1}+\delta_{i 2}, Z_{3}=-2 Z_{1}+\delta_{i 3}, Z_{4}=-Z_{1}+\delta_{i 4}$, and $Z_{6}=-Z_{5}+\delta_{i 6}$, where $\delta_{i k} \sim N(0,0.2)$, independently. The correlation between $Z_{1}$ and each of $Z_{2}, Z_{3}, Z_{4}$ is approximately 0.95 , as is the correlation between $Z_{5}$ and $Z_{6}$.

(N4) $Y_{i}=Z_{1 i}+\ldots+Z_{14 i}+\epsilon_{i}$ (thus, $L=14$ ) and $Z_{2}, \ldots, Z_{10}$ are drawn from a multivariate normal distribution with mean 0 and a covariance matrix with a compound symmetry structure, $0.01 \cdot I_{9}+0.05 \cdot J_{9}$ where $I_{9}$ is a $9 \times 9$ identity matrix and $J_{9}$ is a $9 \times 9$ matrix of 1 's. The average correlation between any pair from the set $Z_{2} \ldots, Z_{10}$ is 0.93 .

(N5) $Y_{i}=Z_{1 i}+\ldots+Z_{20 i}+\epsilon_{i}$ (thus, $L=20$ ) where $Z_{1}, \ldots, Z_{20}$ are drawn from a multivariate normal distribution with an autoregressive (AR1) structure, with $\rho=0.95$.

(N6) $Y_{i}=\sum_{j=1}^{15} \beta_{j} Z_{j i}+\epsilon_{i}$ (thus, $\left.L=15\right)$ where $\beta_{j} \sim N(0,1)$, i.i.d. In this case, the coefficients of the non-null predictors are drawn from the 'slab' component in the spike-andslab model.

(N7) $Y_{i}=\sum_{j=1}^{15} \beta_{j} Z_{j i}+\epsilon_{i}$ (thus, $L=15$ ) where $\beta_{j}=5,1,2,4,9,3,4,1,3,2,4,2,3,1,7$. In this case, the effect sizes have dramatically different magnitudes.

(N8) $Y_{i}=-\sum_{j=1}^{4} \beta_{j} Z_{j i}+\sum_{j=4}^{10} \beta_{j} Z_{j i}+\epsilon_{i}$ (thus, $L=10$ ) where $\beta_{j}=5,7,2,4,9,3,4,1,3,2$. Again, the magnitudes of effect sizes are very different, but this time four of the effects are negative, and six are positive.

(N9) $Y_{i}=-\sum_{j=1}^{4} \beta_{j} Z_{j i}+\sum_{j=4}^{10} \beta_{j} Z_{j i}+\epsilon_{i}$ (thus, $L=10$ ) where $\beta_{j}=2,2,2,2,2,2,6,6,6,6$. Similar setting as the previous scenario in the sense that the magnitudes of effect sizes are very different effects have positive and negative signs, but the effect sizes are from two non-symmetric point masses (unlike our model, which assumes two normal distributions with symmetric means.) 
In our simulations we used different sample sizes and different number of predictors. Results shown here are for $K=1,000$, and $N=100$ (Tables 1 and 2) and $N=50$ (Table 3). The median true positives and false positives were calculated from 30 replications of each scenario. We observe several things:

- For both values of $N$ SEMMS achieves the best or nearly the best results. When $N=100$ it finds all the true predictors in simulations N1-N5 and N7-N8, and no false positive ones in any scenario. When $N=50$, simulations N2-N4 yield a small number of false positives, but the results are still very good.

- In simulation N6 (Table 2) SEMMS finds 7 of the 15 true predictors, but keep in mind that under the spike and slab model which was used to generate the data, a large proportion of the slab component overlaps with the spike component, and it is expected that under this model some true predictors will not be detectable. Note that EMVS which uses the spike and slab model for finding significant predictors yields the same result. In this scenario SIS with a SCAD or MCP penalty achieves a slightly better result.

- When the effect sizes vary dramatically and do not follow our mixture prior assumption, but are not concentrated at non-symmetric mass points (N6-N8), then SEMMS performs very well. When the effect sizes are concentrated at non-symmetric mass points (N9) SEMMS tends to find the largest effect. This is perhaps an unlikely scenario, since we can expect the effect sizes to vary, in which case SEMMS performs very well. In any case, because SEMMS maintains a very low false positive rate, it is possible to deal with cases such as N9 by running SEMMS sequentially, each time moving the detected covariates to the set of 'locked-in' variables until no additional effects are found.

- The spike and slab methods seem very sensitive to deviations from their assumed model. In simulations N7-N9 both EMVS and spikes lab do not detect all the true effects, and have a large number of false positives.

- Perhaps a bit unexpected, in the $N=100$ case the one at a time approach (FDR) performs quite well, especially in scenarios N1, N3, N4, and N5 where it is the second or third best method in terms of its overall error. However, FDR is quite 
conservative in simulations N6-N9, and when $N$ is small relative to $K$ the power of this method is much lower in simulations N2-N4.

- Between the SIS and ncvreg packages which offer similar regularization options the former appears to be better at maintaining lower FP rate.

- Between the two packages that offer a fast Bayesian (spike and slab) fitting, EMVS achieves a lower FP rate in simulations N1-N4 and N6, but higher in simulation N5.

- Generally, both glmnet and lars have high FP rates.

Table 1: Simulation study - normal response, $K=1,000$ predictors, $N=100 . L$ is the true number of predictors used in each scenario.

\begin{tabular}{|l|rr|rr|rr|rr|rr|}
\hline & \multicolumn{2}{|c|}{ Sim. \#N1 } & \multicolumn{2}{|c|}{ Sim. \#N2 } & \multicolumn{2}{c|}{ Sim. \#N3 } & \multicolumn{2}{c|}{ Sim. \#N4 } & \multicolumn{2}{c|}{ Sim. \#N5 } \\
Method & TP & FP & TP & FP & TP & FP & TP & FP & TP & FP \\
\hline SEMMS & 1 & 0 & 8 & 0 & 8 & 0 & 14 & 0 & 20 & 0 \\
ncvreg LASSO & 1 & 5 & 8 & 43.5 & 5 & 17 & 7 & 36 & 8 & 11.5 \\
ncvreg SCAD & 1 & 0 & 8 & 7 & 4 & 1.5 & 6 & 0 & 2 & 15.5 \\
ncvreg MCP & 1 & 0 & 8 & 0.5 & 4 & 0 & 6 & 0 & 2 & 7 \\
glmnet & 1 & 55 & 8 & 31 & 8 & 35 & 14 & 23 & 20 & 33 \\
lars & 1 & 41 & 8 & 21 & 8 & 19 & 11.5 & 0 & 20 & 25.5 \\
SIS LASSO & 1 & 0 & 8 & 8 & 5 & 1 & 7 & 1 & 16 & 5 \\
SIS SCAD & 1 & 0 & 8 & 0 & 4 & 0 & 6 & 0 & 13 & 8 \\
SIS MCP & 1 & 0 & 8 & 0 & 4 & 0 & 6 & 0 & 12.5 & 8.5 \\
EMVS & 1 & 0 & 8 & 0 & 5 & 0 & 6 & 0 & 16.5 & 39 \\
spikeslab & 1 & 0 & 8 & 16 & 6 & 11 & 6 & 5 & 11 & 14 \\
FDR & 1 & 0 & 2.5 & 0 & 7 & 0.5 & 9 & 0 & 20 & 1 \\
\hline
\end{tabular}


Table 2: Simulation study - normal response, $K=1,000$ predictors, $N=100 . L$ is the true number of predictors used in each scenario. In these simulations the true distribution of the significant effects is different from our mixture model.

\begin{tabular}{|l|rr|rr|rr|rr|}
\hline & \multicolumn{2}{|c|}{ Sim. \#N6 } & \multicolumn{2}{|c|}{ Sim. \#N7 } & \multicolumn{2}{|c|}{ Sim. \#N8 } & \multicolumn{2}{c|}{ Sim. \#N9 } \\
Method & TP & FP & \multicolumn{2}{c|}{$L=15$} & \multicolumn{2}{c|}{$L=10$} & \multicolumn{2}{c|}{$L=10$} \\
SEMMS & 7 & 0 & 15 & 0 & 10 & 0 & 4 & 0 \\
ncvreg LASSO & 11 & 30.5 & 15 & 17.5 & 10 & 3 & 10 & 3 \\
ncvreg SCAD & 12 & 11.5 & 15 & 0 & 10 & 0 & 10 & 0 \\
ncvreg MCP & 11 & 5 & 15 & 0 & 10 & 0 & 10 & 0 \\
glmnet & 15 & 85 & 15 & 27 & 10 & 30 & 10 & 27 \\
lars & 15 & 139 & 15 & 4.5 & 10 & 0 & 10 & 1.5 \\
SIS LASSO & 8.5 & 3 & 10 & 11 & 10 & 4 & 10 & 5 \\
SIS SCAD & 8.5 & 0 & 15 & 0 & 10 & 0 & 10 & 0 \\
SIS MCP & 8 & 0 & 15 & 0 & 10 & 0 & 10 & 0 \\
EMVS & 7 & 0 & 9 & 43 & 7 & 40.5 & 7 & 36 \\
spikeslab & 6 & 12.5 & 9 & 14 & 7 & 35 & 7 & 39 \\
FDR & 2 & 0 & 3 & 0 & 3 & 0 & 4 & 0 \\
\hline
\end{tabular}

Table 3: Simulation study - normal response, $K=1,000$ predictors, $N=50 . L$ is the true number of predictors used in each scenario.

\begin{tabular}{|c|c|c|c|c|c|c|c|c|c|c|}
\hline \multirow[b]{2}{*}{ Method } & \multicolumn{2}{|c|}{$\begin{array}{c}\text { Sim. \#N1 } \\
L=1\end{array}$} & \multicolumn{2}{|c|}{$\begin{array}{c}\text { Sim. \#N2 } \\
L=8\end{array}$} & \multicolumn{2}{|c|}{$\begin{array}{c}\text { Sim. \#N3 } \\
L=8\end{array}$} & \multicolumn{2}{|c|}{$\begin{array}{c}\text { Sim. \#N4 } \\
L=14\end{array}$} & \multicolumn{2}{|c|}{$\begin{array}{c}\text { Sim. \#N5 } \\
L=20\end{array}$} \\
\hline & $\mathrm{TP}$ & FP & $\mathrm{TP}$ & FP & $\mathrm{TP}$ & FP & $\mathrm{TP}$ & FP & $\mathrm{TP}$ & $\mathrm{FP}$ \\
\hline SEMMS & 1 & 0 & 2 & 4 & 8 & 2 & 7 & 2.5 & 20 & 0 \\
\hline ncvreg LASSO & 1 & 8.5 & 5.5 & 25 & 5 & 24 & 7 & 30 & 6 & 13.5 \\
\hline ncvreg SCAD & 1 & 0 & 4 & 12.5 & 4 & 11.5 & 6 & 3.5 & 2 & 5 \\
\hline ncvreg MCP & 1 & 0 & 1.5 & 2 & 4 & 4 & 6 & 1 & 1 & 1.5 \\
\hline glmnet & 1 & 45 & 8 & 27 & 8 & 29 & 14 & 21 & 20 & 24 \\
\hline lars & 1 & 22 & 8 & 25 & 8 & 13 & 14 & 10.5 & 20 & 5.5 \\
\hline SIS LASSO & 1 & 0 & 3 & 9 & 4 & 4 & 5.5 & 6 & 11 & 1 \\
\hline SIS SCAD & 1 & 0 & 3 & 9 & 4 & 1 & 6 & 0 & 9 & 3 \\
\hline SIS MCP & 1 & 0 & 2.5 & 8 & 4 & 0 & 6 & 0 & 9.5 & 2.5 \\
\hline EMVS & 1 & 0 & 3 & 5.5 & 5 & 0 & 5 & 0.5 & 16 & 26.5 \\
\hline spikeslab & 0 & 0 & 4 & 25 & 4 & 8.5 & 6 & 13.5 & 9 & 8 \\
\hline FDR & 1 & 0 & 0 & 0 & 2 & 0 & 1 & 0 & 20 & 1.5 \\
\hline
\end{tabular}

We obtained similar results in the binary response case. In Table 4 we show results from two scenarios, both with $K=1000$ and $N=120$. We only use the methods which perform variable selection in the GLM framework, namely, ncvreg, glmnet, SIS, and the one-at-a-time approach, controlling the FDR. The two simulation scenarios are: 
(B1) $\eta_{i}=2 Z_{3 i}+2 Z_{6 i}+2 Z_{7 i}$ but $Z_{2}=Z_{1}+\delta_{i 2}, Z_{3}=-2 Z_{1}+\delta_{i 3}, Z_{4}=-Z_{1}+\delta_{i 4}$, and $Z_{6}=$ $-Z_{5}+\delta_{i 6}$, where $\delta_{i k} \sim N(0,0.2)$, independently. The correlation between $Z_{1}$ and each of $Z_{2}, Z_{3}, Z_{4}$ is approximately 0.95 , as is the correlation between $Z_{5}$ and $Z_{6}$. Thus, in this case $L=7$.

(B2) Autoregressive structure: we set $L=10$ and $\eta_{i}=2 Z_{1, i}+2 Z_{101, i}$ but $Z_{1}-Z_{5}$ have an $\operatorname{AR}(1)$ structure with $\rho=0.95$ and so do $Z_{101}-Z_{105}$.

(B3) Hub network - we create an $N \times K$ matrix so that the columns consist of $g$ nonoverlapping hubs such that within each hub all $\mathrm{K} / \mathrm{g}$ nodes follow a multivariate normal distribution with compound symmetry correlation structure. We pick one node in one of the hubs to be the response, and replace it with a vector of $N 0 / 1$ values, based on a logistic model with the other $L=(K / g)-1$ nodes in that hub as predictors. In Table 4 we show results when $L=9$ (i.e. 100 hubs, each with 10 nodes), but we get very similar with $L=4$ and $L=19$. For this configuration Table 4 includes results with two sample sizes: $N=120$ and $N=80$.

As was the case with the normal response, SEMMS appears to have the best overall performance with the binary responses data. FDR has a slightly higher power in simulation B3 when $N=120$, but it detects zero variables when $N=80$, whereas the performance of SEMMS remains approximately the same when $N$ is decreased from 120 to 80 .

Similarly to the normal and binomial responses, our method has the lowest median false positive rate and a high power to detect the true effects in the Poisson model. Table 4 shows the results of simulation (P1), where $\eta=3+0.3 Z_{1}+0.25 Z_{2}-0.22 Z_{3}-0.19 Z_{4}+$ $0.27 Z_{5}-0.17 Z_{6}-0.25 Z_{7}$ and $N=120$. Simulation (P2) uses the same model, except that predictors $Z_{1}-Z_{5}$ have an $\operatorname{AR}(1)$ structure with $\rho=0.95$. With P1, SEMMS has a median TP five predictors out of seven, and median of 0 false positives. In the P2 setting SEMMS detects all true predictors, while still having no false positives. The competing methods ncvreg, glmnet, S IS all achieve good results in terms of power in P1, detecting all seven predictors (and fewer in P2), but a higher false positive count, as compared with SEMMS. Among ncvreg, glmnet, and SIS the latter appears to yield lower false positive rates, yielding a median of five with all three penalty types in P1. In P2 SIS 
Table 4: Simulation study - binary response (simulations B1 and B2) and the Poisson model (simulation P1), all with $K=1,000$ predictors, $N=120$. For Sim. \#B3 we also show results for $N=80$. $L$ is the true number of predictors used in each scenario.

\begin{tabular}{|c|c|c|c|c|c|c|c|c|c|c|c|c|}
\hline \multirow[b]{2}{*}{ Method } & \multicolumn{2}{|c|}{$\begin{array}{c}\text { Sim. \#B1 } \\
L=7\end{array}$} & \multicolumn{2}{|c|}{$\begin{array}{c}\text { Sim. \#B2 } \\
L=10\end{array}$} & \multicolumn{4}{|c|}{$\begin{array}{c}\text { Sim. \#B3 } \\
L=9(N=12080)\end{array}$} & \multicolumn{2}{|c|}{$\begin{array}{c}\text { Sim. \#P1 } \\
L=7\end{array}$} & \multicolumn{2}{|c|}{$\begin{array}{c}\text { Sim. \#P2 } \\
L=7\end{array}$} \\
\hline & $\mathrm{TP}$ & FP & TP & FP & $\mathrm{TP}$ & $\mathrm{FP}$ & ТP & FP & $\mathrm{TP}$ & FP & $\mathrm{TP}$ & FP \\
\hline SEMMS & 6 & 2 & 7.5 & 3.5 & 5 & 0 & 4 & 1 & 5 & 0 & 7 & 0 \\
\hline ncvreg LASSO & 3.5 & 12.5 & 1.5 & 4.5 & 7 & 23 & 6 & 14 & 7 & 37.5 & 4 & 21.5 \\
\hline ncvreg SCAD & 3 & 12 & 1.5 & 4.5 & 7 & 18 & 5 & 12.5 & 7 & 28 & 3 & 17 \\
\hline ncvreg MCP & 3 & 5.5 & 1 & 0.5 & 5 & 5 & 3 & 3.5 & 7 & 8.5 & 3 & 3.5 \\
\hline glmnet & 7 & 58.5 & 5 & 43.5 & 9 & 53 & 9 & 48 & 7 & 40 & 7 & 47 \\
\hline SIS LASSO & 3 & 2 & 2 & 4 & 4 & 2 & 2 & 2 & 7 & 5 & 5 & 6 \\
\hline SIS SCAD & 3 & 3 & 1 & 4 & 4 & 2 & 2 & 2 & 7 & 5 & 3 & 6.5 \\
\hline SIS MCP & 3 & 3 & 1 & 4 & 4 & 2 & 2 & 2 & 7 & 5 & 3 & 0.5 \\
\hline FDR & 4 & 0 & 0 & 0 & 6 & 0 & 0 & 0 & 3 & 0 & 6 & 0 \\
\hline
\end{tabular}

$\mathrm{MCP}$ has a median of 0.5 false positive, but it detects only three of the seven true predictors. Note that in the one-at-a-time approach, we used the family=quasipoisson () option, and not family=poisson () since the latter yielded a very high false positive rate.

In our simulation results we did not include the two MCMC-based methods (mombf and BoomSpikeSlab) because they proved to be far too slow to be practical, given the existence of fast alternatives, including Bayesian approaches like spikeslab and especially EMVS. For example, when we ran simulations N1 and N5 with momb f but with a much smaller number of predictors $(K=100)$ scenario N1 took an average of 4 minutes to complete each replicate, and N5 took an average of 1.6 hours (with a sample size $N=30$ and 1,000 MCMC iterations in both scenarios.) In the N1 case, the correct model was detected almost every time, but in the case of N5 the median number of true positives was 4 (when $L$ is actually 20), and the median FP was 0 . It appears that momb f gets much slower as $L$ increases, and less powerful as compared with competing methods.

The BoomSpikeSlab package appears to be somewhat faster, taking about 40 minutes to complete 1,000 MCMC iterations when $K=1000$. However, it does not seem to have an advantage over the best available methods in terms of power or error rate. For example, in the case of simulation N5 the median number of predictors found by 
BoomSpikeSlab was 1 (out of 20), with 0 false positives.

\section{Case Studies}

\subsection{Normal Response - the Riboflavin Data}

In a recent paper demonstrating modern approaches to high-dimensional statistics, Bühlmann et al. (2014) analyzed a data set from Lee et al. (2001) in which the response variable is the logarithm of riboflavin (vitamin B12) production rate, and there are normalized expression levels of 4,088 genes which are used as explanatory variables. The sample size in the data set is $N=71$. In addition to the fact that the number of putative variable greatly exceeds the number of observations, many of the putative variables are highly correlated. Out of $8,353,828$ pairs of genes, there are 70,349 with correlation coefficient greater than 0.8 (in absolute value).

Bühlmann et al. (2014) report that the Lasso with $B=500$ independent random subsamples of size $\lfloor N / 2\rfloor$, and with $q=20$ variables that enter the regularization step first, yields three significant and stable genes: LYSC_at, YOAB_at, and YXLD_at. The model with these three variables has an $R^{2}$ of 0.68 , and AIC of 118.6. Their multisample-split method yields one significant variable (YXLD_at), and the projection estimator, used with Ridge-type score yields no significant variables at the FWER-adjusted 5\% significance level. The model with just YXLD_at has an $R^{2}$ of 0.37 and AIC of 162.

Lederer and Müller (2015) also used this data set, to demonstrate their TREX model. Their final model includes three genes: YXLE_at, YOAB_at, and YXLD_at. The $R^{2}$ of their model is 0.62 , and the AIC is 130.68 . Two of the genes are highly correlated (YXLD_at and YXLE_at) and yield a variance inflation factor of 23.7 each, so when fitting the final linear regression model neither appears to be significant.

We ran SEMMS using both the greedy and the weighted probability methods as described in Section 3.2. The greedy method yielded six significant genes: LYSC_at, SPOIISA_at, XTRA_at, YDDK_at, YURQ_at, and YXLD_at. The predicted level of the 

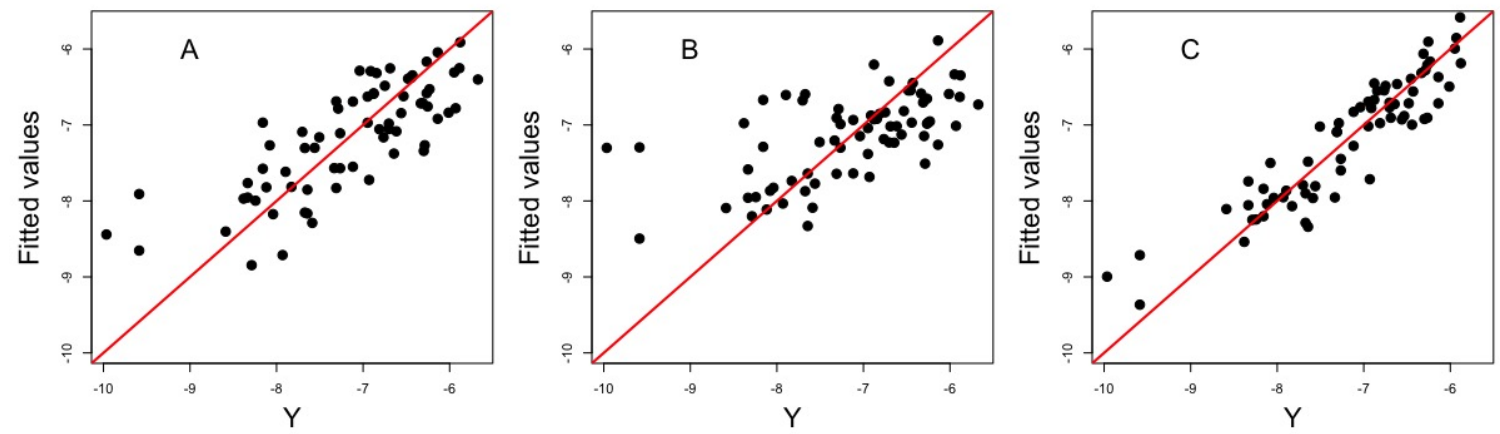

Figure 2: Riboflavin data - fitted vs. observed values. A: Lederer and Müller (2015), B: Bühlmann et al. (2014), C: SEMMS - greedy algorithm.

logarithm of riboflavin production rate is given by the formula:

$$
\begin{aligned}
\hat{Y}= & -7.16-0.23 \cdot Z_{L Y S C \_a t}+0.19 \cdot Z_{S P O I I S A a t}+0.16 \cdot Z_{X T R A \_a t} \\
& -0.23 \cdot Z_{Y D D K \_a t}+0.26 \cdot Z_{Y U R Q \_a t}-0.35 \cdot Z_{Y X L D \_a t}
\end{aligned}
$$

This model has AIC $=70.9$ and an $R^{2}$ of 0.85 . Figure 2 depicts the observed values $(\mathrm{Y})$ vs. the fitted values from three models (Bühlmann et al. (2014), Lederer and Müller (2015), and greedy SEMMS.) In addition to having much smaller residuals than the two other methods, SEMMS provides much better prediction for low values of riboflavin. The other two methods seem to over-estimate the riboflavin levels when the true (normalized) values are small (less than -9 ).

We ran SEMMS using the weighted probability method 100 times. The best model included six genes (CARB_at, SPOVAB_at, XHLA_at, YCKE_at, YOAB_at, YXLD_at) and had an AIC of 53.9 and an $R^{2}$ of 0.88 . In the 100 runs of the randomized SEMMS a total of 16 genes were selected, yielding an AIC of 46.8 and an $R^{2}$ of 0.92 .

We also used packages that performed well in our simulations. EMVS found eight predictors (AIC=68.2) and SIS with MCP penalty found four predictors (AIC=86.3).

The notion of 'the best selected model' may not always be appropriate, since (i) the number of putative variables is large there is no way to evaluate all possible models, and (ii) some selected predictors can be part of a network of highly correlated variables. To 


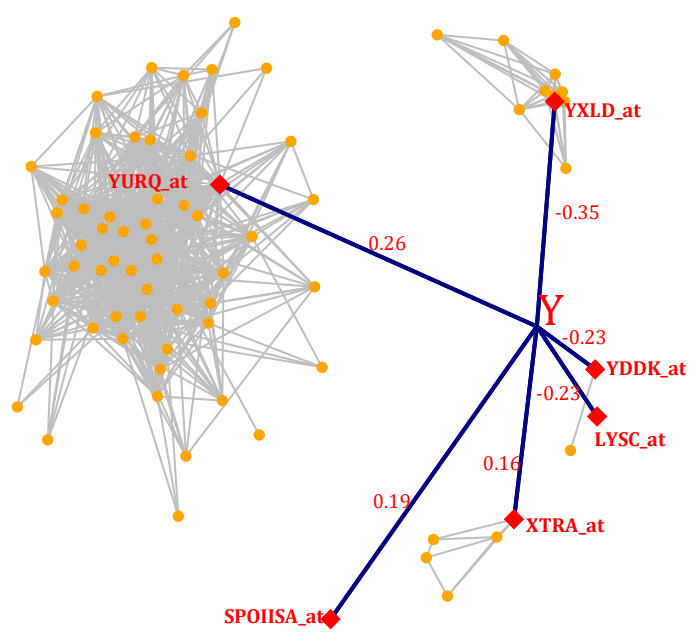

Figure 3: Riboflavin data - a graphical representation of the model found by SEMMS using the greedy algorithm.

deal with (ii) SEMMS detects predictors that are highly correlated with ones selected to be in the model, and reports them as well. This is illustrated in Figure 3 . The variables selected by (greedy) SEMMS are shown as red diamonds and their coefficients in the fitted linear model as numbers next to the (dark blue) edges. Variables that are highly correlated with a selected predictor are depicted as orange dots and they are connected via a gray edge to another predictor if their correlation with that predictor exceeds a user-defined threshold (in this case, we used 0.75). With the riboflavin dataset it can be seen that the YURQ_at gene is co-expressed with a large group of genes, and hence each one of those genes (or perhaps some weighted average of their expression levels) could be considered as a relevant predictor for the response. In this case study, with a correlation threshold of 0.75 between predictors greedy SEMMS reports a total of 62 relevant variables.

\subsection{Binary Response - the BMI Data}

Lin et al.(2014) demonstrated an application of a LASSO-based variable selection method for regression models with compositional covariates. The analysis aims to identify a subset of 87 bacteria genera in the gut whose subcomposition is associated with body- 
mass index (BMI). The data, which was introduced in Wu et al. (2011), is compositional, which using our previous notation means that $\sum_{j=1}^{87} z_{i j}=1$ for each $i$. The total number of samples is $N=96$. To apply our method directly, without changing the model to account for the sum constraint, we perform the $\log$ ratio transformation and replace the matrix $\mathbf{Z}$ with $\mathbf{Z}^{K}=\left[\log \left(z_{i j} / z_{i K}\right)\right]$. The data contains many zero counts, so Lin et al. (2014) replace them with 0.5 before converting the data to be in compositional form. Note that there may be preferable imputation methods, but we choose to use the same method in Lin et al. (2014) in order to have a meaningful comparison. We use a subset of 45 bacteria which had non-zero counts in at least $10 \%$ of the samples $(N=96)$. The omitted genera have minimal contribution to the overall distribution of the proportions.

To demonstrate the application of SEMMS to the binary response case we create an categorical BMI variable with 4 levels: underweight - less than $18.5(n=5)$, normal [18.5, $25)(n=30)$, overweight $[25.5,30)(n=25)$, and obese $\geq 30(n=10)$. We used the binomial option in SEMMS in order to find which bacteria are associated with $p_{b}=\operatorname{Pr}$ (obese) and which are associated with $p_{w}=\operatorname{Pr}$ (overweight), with the normal group as the baseline. The underweight group is too small and is not included in the analysis. We found six bacteria associated with obesity (Acidaminococcus, Alistipes, Allisonella, Butyricimonas, Clostridium, and Oxalobacter), and five with overweight (Anaerofilum, Faecalibacterium, Oscillibacter, Turicibacter, and Veillonella). We also ran SEMMS with BMI as a continuous response, using the normal model and obtained the same four genera reported by Lin et al. (2014) (Acidaminococcus, Alistipes, Allisonella, and Clostridium). The fact that these four are a subset of the genera found to be associated with $p_{b}$ but have no overlap with the ones associated with $p_{w}$ suggest that different BMI levels are associated with different bacteria, and thus, a categorical analysis or perhaps quantile regression may be more appropriate than the conditional mean models in regression analysis.

\subsection{Survival Analysis - the NKI70 data}

Our model can be used to deal with censored survival times. We follow Whitehead (1980) who proposed using an artificial Poisson model to fit Cox's proportional hazards (PH) regression model. Recall that in the proportional hazard model, the hazard func- 
tions have the form $\lambda(t ; \mathbf{z})=\lambda_{0}(t) \exp (\mathbf{z} \boldsymbol{\beta})$. Suppose there are $q$ deaths occurring after survival times $t_{1}, \ldots, t_{q}$ (one death after each $t_{i}$.) For each $h=1, \ldots, q$ let $Y_{h, j}$ be independent Poisson random variables with parameters $\mu_{h, j}$, such that

$$
\mu_{h, j}=N_{h, j} \exp \left(\alpha_{h}+\mathbf{z}_{h, j} \boldsymbol{\beta}\right)
$$

where $N_{h, j}$ is the number of patients who are at risk after $t_{h}$ and whose explanatory vector is $\mathbf{z}_{h, j}\left(t_{h}\right)$. Since the excess life of a patient beyond time $t_{h}-\delta t_{h}$ follows an exponential distribution, the number of deaths in each group follows a Poisson distribution with parameter $\mu_{h, j}$. In other words, rather than modeling the survival times, we model the number of (instantaneous) deaths among the survivors at a given time in a given group. The mean number of instantaneous deaths among survivors in a group of individuals sharing the same properties is associated with a linear combination of the explanatory variables through the log link function. This strategy allows for ties and censoring in the data. Another approach to survival modeling is the accelerated failure time (AFT) models. More biostatisticians are using the AFT model because it is based on the linear model and the estimated regression coefficients have a rather direct physical interpretation. The AFT model can be formulated as variants of estimating equations that correspond to generalized linear models via a Buckley-James transformed Gaussian estimating equations (Buckley and James, 1979).

To demonstrate this approach, we used the NKI70 dataset from van de Vijver et al. (2002), which is available in the 'penalized' package (Goeman, 2010). This dataset contains gene expression measurements for 70 genes, obtained from lymph nodes of 144 breast cancer patients. The 70 genes were determined as prognostic for metastasisfree survival in earlier studies. Large-scale microarray gene expression analyses have revealed a signature set of genes that can predict breast-cancer prognosis van de Vijver et al. (2002), Van't Veer et al. (2002), Wang et al. (2005), Fan et al. (2006), Hua et al. (2008), Madden et al. (2013)). However, few genes overlapped in these assays, and only a few of the breast-associated genes have been validated at the protein level.

We analyzed which genes were associated with either death or recurrence of metastasis in less than three months. We included the (scaled) age of the patients as an explana- 
tory variable, as well as the logarithm of the time until death/recurrence or censoring where, for the purpose of this analysis we considered any individual who survived more than 3 months as censored. SEMMS generated the following model

$$
\begin{array}{r}
\eta=\log \mu=-2.8-0.37 \log (t)-0.35 \cdot Z_{S C U B E 2}-0.79 \cdot Z_{K N T C 2} \\
-0.56 \cdot Z_{Z N F 533}+0.36 \cdot Z_{I G F B P 5.1}+1.09 \cdot Z_{P R C 1}
\end{array}
$$

The residual deviance for this model is 39 (vs. 87.55 for an intercept-only model). These selected genes have been determined to be critical as prognostic for metastasis-free survival in an earlier studies. In particular, a cross-platform comparison of breast-cancer gene sets from these profiling studies revealed SCUBE2 as a common gene that has been validated at the protein level (Lin et al., 2014).

\section{Conclusion}

In 1996 Brad Efron stated that variable selection in regression is the most important problem in statistics (Hesterberg et al. 2008). Since then many papers have been written on the topic, as this continues to be a challenging problem in the age of high-throughput sequencing in genomics, and as other types of 'omics' data become available and more affordable. We have developed a model-based, empirical Bayes approach to variable selection. We define a mixture model in which the putative variables are modeled as random effects, and we demonstrate that this approach results in high power to select the correct variables while maintaining a low rate of false positive selections, in a variety of situations. Our algorithm is scalable and computationally efficient because of: (i) the parsimony of the mixture model, since the mixture model involves a very small number of parameters, and that number remains constant regardless of the number of putative variables; and (ii) the usage of the Generalized Alternating Maximization algorithm to estimate the model parameters, as well as an efficient dimension reduction trick via the Woodbury identity. The Generalized Alternating Maximization algorithm not only converges significantly faster than simulation-based methods, but also uses memory more efficiently, since it has to keep only the posterior non/nonnull probabilities for 
each predictor from only the two latest iterations, as opposed to entire chains. Finally, a simple modification to the algorithm prevents multicollinearity problems in the fitted regression model.

\section{SUPPLEMENTARY MATERIAL}

Title: The file SEMMS_a.pdf contains some implementation notes, instructions how to obtain and install the package, and three examples with code and plots generated by SEMMS. (pdf format)

R-package: version 0.1 .0 of SEMMS is available online at https://haim-bar.uconn. edu/software/. The GNU zipped tar file contains datasets used in the case studies, as well as one simulated dataset and a file with ozone levels data for an additional case study of a well-studied dataset.

\section{A Appendix}

Proof of Proposition 1 We denote the estimates obtained in step $t$ of Algorithm 1 by a superscript. First, we show that the algorithm terminates after a finite number of steps. Given $\boldsymbol{\gamma}^{t}$, the estimate for $\boldsymbol{\theta}$ from Line 3 is unique, because the log-likelihood is a convex function. Therefore, if at any $t, S^{t+1}$ is empty (that is, the loglikelihood will not improve by changing $\gamma$ ) then the algorithm terminates because the estimate for $\boldsymbol{\theta}$ will not change in the subsequent iteration. So, we need to show that for all $t \geq 0$ and $s>1$ we cannot have $\gamma^{t}=\gamma^{t+s}$ (i.e., there are no loops in the sequence of $\gamma^{t}$ ). Suppose that for some $t$ there exists $s>1$ such that $\gamma^{t}=\gamma^{t+s}$. Then, the convexity of the loglikelihood function implies that $\boldsymbol{\theta}^{t}=\boldsymbol{\theta}^{t+s}$. Together, this means that $\ell^{t}(\mathbf{y})=\ell^{t+s}(\mathbf{y})$, but in the algorithm we selected the coordinates to modify in each step, $k_{t}, \ldots, k_{t+s}$, so that in each iteration the log-likelihood strictly increased, so we must have $\ell^{t+s}(\mathbf{y})-\ell^{t}(\mathbf{y})>0$. Proposition 7 in Gunawardana and Byrne (2005) shows that minimization (and consequently maximization) of a continuous function forms a closed point-to-set map. Their Lemma 9 shows the Cartesian product of two closed point-to-set-maps is itself closed. With these results in hand one can apply Zangwill's generalized convergence theorem (Zangwill, 1969) to the sequence $\left(\boldsymbol{\theta}^{t}, \boldsymbol{\gamma}^{t}\right)$. 


\section{References}

Bai, R. and M. Ghosh (2017). The inverse gamma-gamma prior for optimal posterior contraction and multiple hypothesis testing. arXiv preprint arXiv:1710.04369.

Benjamini, Y. and Y. Gavrilov $(2009,03)$. A simple forward selection procedure based on false discovery rate control. Annals of Applied Statistics 3(1), 179-198.

Benjamini, Y. and Y. Hochberg (1995). Controlling the false discovery rate-a practical and powerful approach to multiple testing. Journal of The Royal Statistical Society Series B 57(3), 499-517.

Bhadra, A., J. Datta, N. G. Polson, B. Willard, et al. (2017). The horseshoe+ estimator of ultra-sparse signals. Bayesian Analysis 12(4), 1105-1131.

Bogdan, M., E. van den Berg, C. Sabatti, W. Su, and E. Candes (2014). Slope - adaptive variable selection via convex optimization. arXiv:1407.3824.

Breheny, P. and J. Huang (2011). Coordinate descent algorithms for nonconvex penalized regression, with applications to biological feature selection. Annals of Applied Statistics 5(1), 232-253.

Breslow, N. and M. Clayton (1993). Approximate inference in generalized linear models. Journal of the American Statistical Association 88, 9-25.

Buckley, J. and I. James (1979). Linear regression with censored data. Biometrika 66, 429-436.

Bühlmann, P., M. Kalisch, and L. Meier (2014). High-dimensional statistics with a view toward applications in biology. Annual Review of Statistics and Its Application 1(1), 255278.

Carvalho, C. M., N. G. Polson, and J. G. Scott (2010). The horseshoe estimator for sparse signals. Biometrika 97(2), 465-480.

Casella, G. and E. Moreno (2006). Objective Bayesian variable selection. Journal of the American Statistical Association 101, 157-167. 
Chen, J. and P. Li (2009). Hypothesis test for normal mixture models: The EM approach. Annals of Statistics 37(5A), 2523-2542.

Dempster, A. P., N. M. Laird, and D. B. Rubin (1977). Maximum likelihood from incomplete data via the EM algorithm. Journal of The Royal Statistical Society, Series B 39(1), $1-38$.

Efron, B., T. Hastie, I. Johnstone, and R. Tibshirani (2004). Least angle regression. Annals of Statistics 32, 407-499.

Fan, C., D. S. Oh, L. Wessels, B. Weigelt, D. S. Nuyten, A. B. Nobel, L. J. Van't Veer, and C. M. Perou (2006). Concordance among gene-expression-based predictors for breast cancer. New England Journal of Medicine 355(6), 560-569.

Fan, J. and R. Li (2001). Variable selection via nonconcave penalized likelihood and its oracle properties. Journal of the American Statistical Association 96, 1348-1360.

Friedman, J., T. Hastie, and R. Tibshirani (2010). Regularization paths for generalized linear models via coordinate descent. Journal of Statistical Software 33(1), 1-22.

George, E. and R. McCulloch (1993). Variable selection via Gibbs sampling. Journal of the American Statistical Association 88, 881-889.

Goeman, J. J. (2010). L1 penalized estimation in the cox proportional hazards model. Biometrical Journal (52), -14 .

Golub, G. and C. Van Loan (1996). Matrix Computations. Baltimore, MD, US: The Johns Hopkins University Press.

Guan, Y. and M. Stephens (2012). Bayesian variable selection regression for genomewide association studies and other large-scale problems. Annals of Applied Statistics 5(3), 1780-1815.

Gunawardana, A. and W. Byrne (2005, December). Convergence theorems for generalized alternating minimization procedures. J. Mach. Learn. Res. 6, 2049-2073. 
Hahn, P. R. and C. M. Carvalho (2015). Decoupling shrinkage and selection in bayesian linear models: a posterior summary perspective. Journal of the American Statistical Association 110(509), 435-448.

Hastie, T. and B. Efron (2013). lars: Least Angle Regression, Lasso and Forward Stagewise. R package version 1.2.

Hesterberg, T. C., N. H. Choi, L. Meier, and C. Fraley (2008). Least angle and L1 penalized regression: A review. Statistics Surveys 2, 61-93.

Hocking, R. R. (1976). The analysis and selection of variables in linear regression. Biometrics 32, 661-675.

Hua, S., C. B. Kallen, R. Dhar, M. T. Baquero, C. E. Mason, B. A. Russell, P. K. Shah, J. Liu, A. Khramtsov, M. S. Tretiakova, et al. (2008). Genomic analysis of estrogen cascade reveals histone variant h2a. $\mathrm{z}$ associated with breast cancer progression. Molecular systems biology 4(1), 188.

Ishwaran, H., J. Rao, and U. Kogalur (2013). Prediction and variable selection using spike and slab regression. $\mathrm{R}$ package version 1.1.5.

Ishwaran, H. and J. S. Rao (2005). Spike and slab variable selection: Frequentist and Bayesian strategies. Annals of Statistics 33(2), 730-773.

Johnson, V. E. and D. Rossell (2010). On the use of non-local prior densities in bayesian hypothesis tests. Journal of the Royal Statistical Society: Series B (Statistical Methodology) 72(2), 143-170.

Johnson, V. E. and D. Rossell (2012). Bayesian model selection in high-dimensional settings. Journal of the American Statistical Association 107(498), 649-660.

Lederer, J. and C. Müller (2015). Don't fall for tuning parameters: Tuning-free variable selection in high dimensions with the TREX. In Proceedings of the Twenty-Ninth AAAI Conference on Artificial Intelligence, January 25-30, 2015, Austin, Texas, USA., pp. 27292735. 
Lee, J.-M., S. Zhang, S. Saha, S. Santa Anna, C. Jiang, and J. Perkins (2001). Rna expression analysis using an antisensebacillus subtilis genome array. Journal of Bacteriology 183(24), 7371-7380.

Lin, W., P. Shi, R. Feng, and H. Li (2014). Variable selection in regression with compositional covariates. Biometrika 101(4), 785-797.

Lin, Y.-C., Y.-C. Lee, L.-H. Li, C.-J. Cheng, and R.-B. Yang (2014). Tumor suppressor scube2 inhibits breast-cancer cell migration and invasion through the reversal of epithelial-mesenchymal transition. J Cell Sci 127(1), 85-100.

Lockhart, R., J. Taylor, R. Tibshirani, and R. Tibshirani (2013). covTest: Computes covariance test for adaptive linear modelling. $\mathrm{R}$ package version 1.02 .

Madden, S. F., C. Clarke, P. Gaule, S. T. Aherne, N. O’Donovan, M. Clynes, J. Crown, and W. M. Gallagher (2013). Breastmark: an integrated approach to mining publicly available transcriptomic datasets relating to breast cancer outcome. Breast Cancer Research 15(4), R52.

McGilchrist, C. (1994). Estimation in generalized mixed models. Journal of the Royal Statistical Society B 56, 61-69.

McLachlan, G. J. and D. Peel (2000). Finite mixture models. New York: Wiley Series in Probability and Statistics.

Park, T. and G. Casella (2008). The bayesian lasso. Journal of the American Statistical Association 103(482), 681-686.

Rossell, D., J. D. Cook, D. Telesca, and P. Roebuck (2018). mombf: Moment and Inverse Moment Bayes Factors. R package version 2.1.1.

Rǒcková, V. and E. I. George (2014). EMVS: The EM approach to Bayesian variable selection. Journal of the American Statistical Association 109(506), 828-846.

Rǒcková, V. and G. Moran (2018). EMVS: The Expectation-Maximization Approach to Bayesian Variable Selection. R package version 1.0. 
Saldana, D. F. and Y. Feng (2018). SIS: An R package for sure independence screening in ultrahigh-dimensional statistical models. Journal of Statistical Software 83(2), 1-25.

Schall, R. (1991). Estimation in generalized linear models with random effects. Biometrika 78, 719-727.

Scott, S. L. (2017). BoomSpikeSlab: MCMC for Spike and Slab Regression. R package version 0.9 .0 .

Searle, S. R., G. Casella, and C. E. McCulloch (1992). Variance Components. New York: Wiley-Interscience.

Tibshirani, R. (1996). Regression shrinkage and selection via the lasso. Journal of the Royal Statististical Society, Series B 58(1), 267-288.

van de Vijver, M. J., Y. D. He, L. J. van 't Veer, H. Dai, A. A. Hart, D. W. Voskuil, G. J. Schreiber, J. L. Peterse, C. Roberts, M. J. Marton, M. Parrish, D. Atsma, A. Witteveen, A. Glas, L. Delahaye, T. van der Velde, H. Bartelink, S. Rodenhuis, E. T. Rutgers, S. H. Friend, and R. Bernards (2002). A gene-expression signature as a predictor of survival in breast cancer. New England Journal of Medicine 347(25), 1999-2009. PMID: 12490681.

van der Pas, S., J.-B. Salomond, J. Schmidt-Hieber, et al. (2016). Conditions for posterior contraction in the sparse normal means problem. Electronic Journal of Statistics 10(1), 976-1000.

Van't Veer, L. J., H. Dai, M. J. Van De Vijver, Y. D. He, A. A. Hart, M. Mao, H. L. Peterse, K. Van Der Kooy, M. J. Marton, A. T. Witteveen, et al. (2002). Gene expression profiling predicts clinical outcome of breast cancer. Nature 415(6871), 530-536.

Wang, Y., J. G. Klijn, Y. Zhang, A. M. Sieuwerts, M. P. Look, F. Yang, D. Talantov, M. Timmermans, M. E. Meijer-van Gelder, J. Yu, et al. (2005). Gene-expression profiles to predict distant metastasis of lymph-node-negative primary breast cancer. The Lancet 365(9460), 671-679.

Whitehead, J. (1980). Fitting Cox's regression model to survival data using glim. Applied Statistics 29, 268-275. 
Wolfinger, R. and M. O'Connell (1993). Generalized linear mixed models: a pseudolikelihood approach. Journal of Statistical Computation and Simulation 48, 233-243.

Wu, C. F. J. $(1983,03)$. On the convergence properties of the EM algorithm. Annals of Statistics 11(1), 95-103.

Wu, G. D., J. Chen, C. Hoffmann, K. Bittinger, Y.-Y. Chen, S. A. Keilbaugh, M. Bewtra, D. Knights, W. A. Walters, R. Knight, et al. (2011). Linking long-term dietary patterns with gut microbial enterotypes. Science 334, 105-108.

Zangwill, W. I. (1969). Nonlinear programming : a unified approach. Englewood Cliffs, N.J.: Prentice-Hall. Bibliography: p. 332-345.

Zhang, C.-H. (2010). Nearly unbiased variable selection under minimax concave penalty. Annals of Statistics 38(2), 894-942.

Zhang, M., K. L. Montooth, M. T. Wells, A. G. Clark, and D. Zhang (2005). Mapping multiple quantitative trait loci by Bayesian classification. Genetics 169, 2305-2318.

Zou, H. (2006). The adaptive lasso and its oracle properties. Journal of the American Statistical Association 101(476), 1418-1429. 